

\title{
Non-linear normal modes (nnms) and their applications in vibration theory: an overview
}

\author{
Alexander Vakakis
}

\section{To cite this version:}

Alexander Vakakis. Non-linear normal modes (nnms) and their applications in vibration theory: an overview. Mechanical Systems and Signal Processing, 1997, 11 (1), pp.3-22. 10.1006/mssp.1996.9999 . hal-01354037

\section{HAL Id: hal-01354037 \\ https://hal.science/hal-01354037}

Submitted on 17 Aug 2016

HAL is a multi-disciplinary open access archive for the deposit and dissemination of scientific research documents, whether they are published or not. The documents may come from teaching and research institutions in France or abroad, or from public or private research centers.
L'archive ouverte pluridisciplinaire HAL, est destinée au dépôt et à la diffusion de documents scientifiques de niveau recherche, publiés ou non, émanant des établissements d'enseignement et de recherche français ou étrangers, des laboratoires publics ou privés. 


\title{
NON-LINEAR NORMAL MODES (NNMs) AND THEIR APPLICATIONS IN VIBRATION THEORY: AN OVERVIEW
}

\author{
A. F. VAKAKIS \\ Department of Mechanical and Industrial Engineering, University of Illinois at \\ Urbana-Champaign, 1206 W, Green Street, Urbana, IL 61801, U.S.A.
}

\begin{abstract}
The concept of 'non-linear normal mode' (NNM) is discussed. After providing some introductory definitions, the applications of NNMs to vibration theory are considered. In particular, it is shown how this concept can be used to study forced resonances of non-linear systems and non-linear localisation of vibrational energy in symmetric systems. NNMs can provide a valuable tool for understanding certain essentially non-linear dynamic phenomena that have no counterparts in linear theory and that cannot be analysed by conventional linearised methods. Additional applications of NNMs to modal analysis, model reduction, vibration and shock isolation designs, and the theory of non-linear oscillators are also discussed.
\end{abstract}

\section{INTRODUCTION}

The title of this paper might seem self-contradictory: engineers and physicists traditionally associate the concept of 'normal mode' with linear vibration theory and regard it as closely related to the principle of linear superposition. Indeed, a classical result of linear theory is that the modes of vibration of a discrete or continuous system can be used to decouple the equations of motion, and to express arbitrary free or forced oscillations as superpositions of modal responses. Moreover, in linear theory the number of normal modes of vibration cannot exceed the number of degrees of freedom (dof) of the system, and any forced resonances of the system under external harmonic forces always occur in neighborhoods of normal modes. On the other hand, it is a well-known result that the principle of linear superposition does not apply in non-linear systems. Thus, the following questions arise: can the concept of 'normal mode' be extended to non-linear theory; what is the need for such an extension; and what are the potential applications of such a concept in vibration theory?

The principal aim of this paper is to address these questions. In addition, to discussing the concept of non-linear normal mode (NNM), it is attempted to show how this concept can be used to better understand the free and forced dynamics of non-linear oscillators. Furthermore, the similarities and dissimilarities between linear and non-linear normal modes are examined, and it is shown that NNMs can provide a valuable theoretical tool for understanding certain dynamic phenomena that have no counterparts in linear theory, 
such as mode bifurcations and non-linear mode localisation. Such phenomena cannot be modeled by standard linearised techniques.

\section{DEFINITION OF AN NNM}

A first and obvious definition of an NNM is a non-linear extension of the definition of the normal mode of classical vibration theory. In that context, one defines an NNM of an undamped discrete or continuous system as a synchronous periodic oscillation where all material points of the system reach their extreme values or pass through zero simultaneously. Clearly, when a discrete system vibrates in an NNM, the corresponding oscillation is represented by a line in its configuration space, which is termed modal line. A modal line represents the synchronous oscillation of the system in the configuration space during an NNM motion. Linear systems possess straight modal lines since their co-ordinates are related linearly during a normal mode oscillation. In non-linear systems, the modal lines can be either straight or curved. The later cases are generic in non-linear discrete systems, since straight non-linear modal lines reflect symmetries of the system $[1,2]$.

Based on the previous definition for NNMs, Lyapunov [3] proved the existence of $n$ synchronous periodic solutions (NNMs) in neighborhoods of stable equilibrium points of $n$ dof Hamiltonian systems with no internal resonances, i.e. with linearised eigenfrequencies not integrably related. Weinstein [4] and Moser [5] extended Lyapunov's result to systems with internal resonances.

Constructive techniques for computing NNMs were given by Kauderer [6] and Rosenberg and co-workers, who developed general qualitative [7], and quantitative [8-14] techniques for analysing NNMs in discrete conservative oscillators. For discrete systems Rosenberg defined as similar NNMs that correspond to straight modal lines in configuration space, and as non-similar NNMs that correspond to modal curves (Fig. 1). As mentioned previously, a non-linear discrete system is expected to possess non-similar NNMs, in contrast to the linear case where all modes are similar. Some additional quantitative techniques based on the previous definition of NNMs were performed in [1, 15-33].

The concepts of similar and non-similar NNMs are now demonstrated by means of two simple examples. First, the 2-dof symmetric non-linear system of Fig. 2 is considered with governing equations of motion given by [2]:

$$
\begin{aligned}
& \ddot{x}_{1}+x_{1}+x_{1}^{3}+K\left(x_{1}-x_{2}\right)^{3}=0 \\
& \ddot{x}_{2}+x_{2}+x_{2}^{3}-K\left(x_{1}-x_{2}\right)^{3}=0
\end{aligned}
$$

where cubic stiffness non-linearities are assumed. This system possesses similar NNMs, corresponding to the following linear relation between the depended variables $x_{1}$ and $x_{2}$ :

$$
x_{2}(t)=c x_{1}(t)
$$

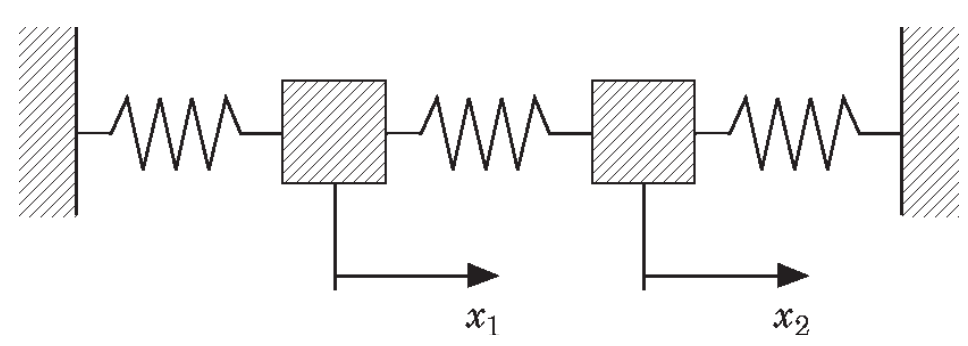

Figure 1. The 2-dof non-linear oscillator. 
linear system (which has as many modes as dof), and thus, the bifurcating NNMs are essentially non-linear motions and cannot be regarded as analytic continuations of any linear modes. This is in contrast to the modes $c= \pm 1$ which can be regarded as non-linear continuations of the normal modes of the linear system. This simple example shows that the normal modes of a non-linear system may exceed in number its dof, a feature which is in contrast to the predictions of linear theory. Moreover, NNMs can be either stable or unstable [1], a feature which is in contrast to linear theory where all modes are neutrally stable. By mode instability, we mean that small perturbations of the initial conditions that are required for the realisation of the NNM lead to the elimination of the mode oscillation.

As pointed out previously [1,2], similar NNMs are not generic in non-linear systems since they require special symmetry conditions (note that the system of Fig. 2 is symmetrical). Generically, NNMs are non-similar, and (unfortunately) more difficult to compute. Here, the concept of non-similar NNM is demonstrated by examining the system with the configuration of Fig. 2 but with non-symmetric grounding stiffnesses. The equations of motion are given by [34],

$$
\begin{gathered}
\ddot{x}_{1}+x_{1}+v_{1} x_{1}^{3}+k_{1}\left(x_{1}-x_{2}\right)+v_{2}\left(x_{1}-x_{2}\right)^{3}=0 \\
\ddot{x}_{2}+\left(1+\alpha_{1}\right) x_{2}+v_{1}\left(1+\alpha_{3}\right) x_{2}^{3}+k_{1}\left(x_{2}-x_{1}\right)+v_{2}\left(x_{2}-x_{1}\right)^{3}=0
\end{gathered}
$$

coupled with initial conditions,

$$
x_{1}(0)=X_{1}, \quad \dot{x}_{1}(0)=0, \quad x_{2}(0)=X_{2}, \quad \dot{x}_{2}(0)=0
$$

The scalars $v_{1}$ and $v_{2}$ are the non-linear stiffness terms of the grounding and coupling stiffnesses, respectively, and $\alpha_{1}, \alpha_{3}$ are mistuning parameters adjusting the asymmetry of the system. When $\alpha_{1} \alpha_{2} \neq 0$, the symmetry of the system is perturbed, and the previously computed similar NNMs can no longer exist. At this point we assume weak coupling between the two masses by rescaling the coupling stiffness coefficients as, $k_{1}=\varepsilon K_{1}, v_{2}=\varepsilon N_{2}$, where $\varepsilon$ is a small parameter, $|\varepsilon| \ll 1$. A non-similar NNM of the oscillator (5) is sought in the form [compare with the requirement for similar NNM, equation (2)]:

$$
x_{2}=\hat{x}_{2}\left(x_{1}\right)
$$

where the modal function $\hat{x}_{2}(\cdot)$ governs the trajectory of the mode in the configuration plane $\left(x_{1}, x_{2}\right)$. Since (6) must hold at every instant of time, the derivatives of the positional variables for motion on a non-similar NNM can be expressed by the chain rule as follows:

$$
\dot{x}_{2}=\hat{x}_{2}^{\prime} \dot{x}_{1}, \quad \ddot{x}_{2}=\hat{x}_{2}^{\prime \prime}\left(\dot{x}_{1}\right)^{2}+\hat{x}_{2}^{\prime} \ddot{x}_{1}
$$

where $(\cdot) \equiv \mathrm{d} / \mathrm{d} x_{1}$. Substituting for $x_{2}, \dot{x}_{2}$, and $\ddot{x}_{2}$ into the equations of motion (5), one obtains the following alternative set of equations of motion that describe the oscillation on the non-similar mode:

$$
\begin{gathered}
\ddot{x}_{1}+x_{1}+v_{1} x_{1}^{3}+\varepsilon K_{1}\left(x_{1}-\hat{x}_{2}\right)+\varepsilon N_{2}\left(x_{1}-\hat{x}_{2}\right)^{3}=0 \\
\hat{x}_{2}^{\prime \prime}\left(\dot{x}_{1}\right)^{2}+\hat{x}_{2}^{\prime} \ddot{x}_{1}+\left(1+\alpha_{1}\right) \hat{x}_{2}+v_{1}\left(1+\alpha_{3}\right) \hat{x}_{2}^{3}+\varepsilon K_{1}\left(\hat{x}_{2}-x_{1}\right)+\varepsilon N_{2}\left(\hat{x}_{2}-x_{1}\right)^{3}=0
\end{gathered}
$$

An expression for the velocity $\dot{x}_{1}$ can be obtained by integrating the first of the above equations:

$$
\left(\dot{x}_{1}\right)^{2}=-2 \int_{X_{1}}^{x_{1}}\left\{\xi\left(1+\varepsilon K_{1}\right)+v_{1} \xi^{3}-\varepsilon K_{1} \hat{x}_{2}(\xi)+\varepsilon N_{2}\left[\xi-\hat{x}_{2}(\xi)\right]^{3}\right\} \mathrm{d} \xi
$$


where $X_{1}$ is the maximum amplitude attained by the parametrising co-ordinate $x_{1}$. Substituting (9) into the second of equations (8) and eliminating $\ddot{x}_{1}$, the following differential equation is obtained governing the modal function $\hat{x}_{2}(\cdot)$ :

$$
\begin{aligned}
-2 \hat{x}_{2}^{\prime \prime}[ & \frac{\left(x_{1}^{2}+X_{1}^{2}\right)}{2}\left(1+\varepsilon K_{1}\right)+v_{1} \frac{\left(x_{1}^{4}-X_{1}^{4}\right)}{4} \\
& \left.+\int_{X_{1}}^{x_{1}}\left\{\varepsilon N_{2}\left[\xi-\hat{x}_{2}(\xi)\right]^{3}-\varepsilon K_{1} \hat{x}_{2}(\xi)\right\} \mathrm{d} \xi\right] \\
& -\hat{x}_{2}^{\prime}\left[x_{1}+v_{1} x_{1}^{3}+\varepsilon K_{1} x_{1}-\varepsilon \hat{x}_{2} K_{1}+\varepsilon N_{2}\left(x_{1}-\hat{x}_{2}\right)^{3}\right] \\
& +\left(1+\alpha_{1}\right) \hat{x}_{2}+v_{1}\left(1+\alpha_{3}\right) \hat{x}_{2}^{3}+\varepsilon K_{1} \hat{x}_{2}-\varepsilon K_{1} x_{1}+\varepsilon N_{2}\left(\hat{x}_{2}-x_{1}\right)^{3}=0
\end{aligned}
$$

The non-similar NNMs of the problem are solutions of (10) and are computed using successive approximations of the following asymptotic form [34]:

$$
\hat{x}_{2}\left(x_{1}\right)=\hat{x}_{2}^{(0)}\left(x_{1}\right)+\epsilon \hat{x}_{2}^{(1)}\left(x_{1}\right)+\cdots
$$

The details of the computation are omitted and the interested reader is referred to [1,34] (for an exact non-similar NNM solution see [35]). Note that, in contrast to similar NNMs which do not depend on the level of the (conserved) energy of the oscillation, non-similar NNMs are energy-dependent; that is, the expression for the modal function (6) depends on the actual energy of oscillation, a feature that complicates the calculation of non-similar NNM trajectories. Figure 3 presents schematically similar and non-similar NNM trajectories in the configuration space $\left(x_{1}, x_{2}\right)$. The ellipsoid enclosing the curved modal line represents the surface of maximum potential energy of the system reached when $x_{1}=X_{1}$, i.e. when the parametrised variable $x_{1}$ attains its maximum value. Since a NNM corresponds to synchronous oscillations of all co-ordinates of the system, when $x_{1}=X_{1}$ all the other co-ordinates reach their maximum values and the potential energy of the oscillator is maximised.

Now, the existence of NNMs in non-conservative systems with damping are discussed. To study free non-linear oscillations in this class of systems, an extension of the previous NNM definition is introduced based on the concept of invariant manifold. This extended definition is necessary since the free oscillation of a damped system is not synchronous, and non-trivial phase differences are expected between the positional variables. Previously, the concept of NNM for a general class of non-linear discrete oscillators has been reformulated [25-27], without assuming the existence of an analytic first integral of motion. This analysis was carried out in the real domain (real invariant manifold formulation) and was based on the computation of invariant manifolds of motion on which the NNM oscillations take place. The parametrisation of the invariant manifolds of the NNMs was performed by employing two independent reference variables, namely, a reference positional displacement and a reference positional velocity. A computationally efficient extension of the invariant manifold methodology was proposed by [31] who reformulated the invariant manifold method on a complex framework (complex invariant manifold formulation). A further advantage of the complex approach is that it can be easily extended to compute higher-dimensional invariant manifolds of NNMs in internal resonance [36].

The real invariant manifold formulation is demonstrated by considering the $n$-dof non-linear autonomous oscillator:

$$
\ddot{x}_{i}+f_{i}(x, \dot{x})=0, \quad i=1,2, \ldots, n
$$


where $x$ denotes the $(n \times 1)$ vector of positional variables, and $f_{i}(x, \dot{x})$ smooth non-linearities. First, (12) is expressed in state form as follows:

$$
\dot{x}_{i}=y_{i} \quad \dot{y}_{i}=-f_{i}(x, y), \quad i=1,2, \ldots, n
$$

Equations (12) or (13) possess a NNM if there exists a motion for which all displacements and velocities can be parametrised by a single reference displacement-velocity pair, say $\left(x_{1}, y_{1}\right) \equiv(x, y)$ :

$$
x_{i}=X_{i}(x, y), \quad y_{i}=Y_{i}(x, y), \quad i=2, \ldots, n
$$

The relations (14) define a constraint 2-D surface in the $2 n$-D phase space of the dynamical system which is defined as the 2-D NNM invariant manifold. The NNM invariant manifold is computed as previously, that is, using (14) to eliminate the explicit time dependence from (13) and formulating a set of differential equations governing the functions $X_{i}(x, y)$ and $Y_{i}(x, y), i=2, \ldots, n$. Then, the following set of differential equations governing these functions are obtained:

$$
\begin{aligned}
& Y_{i}-\frac{\partial X_{i}}{\partial x} y=\frac{\partial X_{i}}{\partial y} f_{1}\left(x, X_{2}, \ldots, X_{n}, y, Y_{2}, \ldots, Y_{n}\right) \\
& \frac{\partial Y_{i}}{\partial x} y+\frac{\partial Y_{i}}{\partial y} f_{1}\left(x, X_{2}, \ldots, X_{n}, y, Y_{2}, \ldots, Y_{n}\right) \\
& \quad=f_{i}\left(x, X_{2}, \ldots, X_{n}, y, Y_{2}, \ldots, Y_{n}\right), \quad i=2, \ldots n
\end{aligned}
$$

Once a solution for $X_{i}(x, y)$ and $Y_{i}(x, y), i=2, \ldots, n$, is derived, the time response of the dynamical system is computed by expressing the first pair of equations (13) in the form:

$$
\begin{aligned}
& \dot{x}=y, \dot{y}=-f_{1}\left[x, X_{2}(x, y), \ldots, X_{n}(x, y), y, Y_{2}(x, y), \ldots, Y_{n}(x, y)\right] \\
& \Rightarrow \quad \ddot{x}+f_{1}\left[x, X_{2}(x, \dot{x}), \ldots, X_{n}(x, \dot{x}), \dot{x}, Y_{2}(x, \dot{x}), \ldots, Y_{n}(x, \dot{x})\right]=0
\end{aligned}
$$

and solving these ordinary differential equations to determine $x(t)$ and $y(t)$ using well-established non-linear techniques such as the method of averaging or the method of multiple scales.

The partial differential equations (15) are, in principle, as difficult to solve as the original problem (12). However, an approximate solution can be developed using power series expansions as follows [27]:

$$
\begin{aligned}
X_{i}(x, y)= & a_{i}^{(0)}+a_{i}^{(1)} x+a_{i}^{(2)} y+a_{i}^{(3)} x^{2}+a_{i}^{(4)} y^{2}+a_{i}^{(5)} x y \\
& +a_{i}^{(6)} x^{3}+a_{i}^{(7)} y^{3}+a_{i}^{(8)} x^{2} y+a_{i}^{(9)} x y^{2}+\cdots \\
Y_{i}(x, y)= & b_{i}^{(0)}+b_{i}^{(1)} x+b_{i}^{(2)} y+b_{i}^{(3)} x^{2}+b_{i}^{(4)} y^{2}+b_{i}^{(5)} x y \\
& \quad+b_{i}^{(6)} x^{3}+b_{i}^{(7)} y^{3}+b_{i}^{(8)} x^{2} y+b_{i}^{(9)} x y^{2}+\cdots, \quad i=2, \ldots, n
\end{aligned}
$$

Substituting (17) into (15) and matching the coefficients of the monomials $\left(x^{m} y^{n}\right)$ one derives a set of coupled algebraic equations in terms of the coefficients $a_{i}^{(p)}$ and $b_{i}^{(q)}$, which can be solved using standard linear algebra techniques.

Additional definitions of NNMs were formulated in the literature, including group theoretic definitions based on discrete and continuous groups [2]; however, since these approaches are involved theoretically they are not reviewed in this work. In an additional series of works [37-42] special techniques for analysing the NNMs (and their bifurcations) of non-linear continuous systems, such as systems of beams and rods, are discussed. 


\section{APPLICATIONS OF NNMs}

The previous section showed how the concept of normal mode can be extended to non-linear systems. This section will discuss how NNMs can be used to study and understand better the dynamics of mechanical oscillators. As discussed in the previous section, a discrete non-linear system can possess more normal modes than its dof, since a subset of NNMs can be essentially non-linear with no counterparts in linear theory. Hence, extending linear concepts such as modal analysis to non-linear systems must be performed with care [for example, a non-linear system with 2 dof may possess more than two normal modes (Fig. 2)]. In the following, two main applications of NNMs are discussed in detail, namely, their influence on forced resonances and non-linear localisation and motion confinement. Later, new potential areas of applications of NNMs to vibration analysis are summarised.

\subsection{STUDY OF FORCED RESONANCES}

An important property of NNMs relates to forced resonances. In analogy to linear theory, forced resonances in non-linear systems occur in neighborhoods of NNMs [2]. Hence, knowledge of the structure of the normal modes of a non-linear oscillator can provide valuable insight on the structure of its resonances, a feature of considerable engineering importance. Moreover, since the number of normal modes of a non-linear oscillator may exceed its dof, certain forced resonances are essentially non-linear and have no analogies in linear theory; in such cases a 'linearisation' of the system either might not be possible, or might not provide all the possible resonances that can be realised.

The above points can be demonstrated by means of two examples. First, consider the symmetric system of Fig. 1 with viscous damping and forced by harmonic excitations. A detailed analysis of this system was performed in [43] and only a summary of results is provided here. The equations of motion are given by:

$$
\begin{aligned}
& \ddot{x}_{1}+x_{1}+\varepsilon c_{1} \dot{x}_{1}+\varepsilon x_{1}^{3}+\varepsilon K\left(x_{1}-x_{2}\right)^{3}=2 \varepsilon P_{1} \cos \omega t \\
& \ddot{x}_{2}+x_{2}+\varepsilon c_{2} \dot{x}_{2}+\varepsilon x_{2}^{3}+\varepsilon K\left(x_{2}-x_{1}\right)^{3}=2 \varepsilon P_{2} \cos \omega t
\end{aligned}
$$

It is assumed that the damping, the coupling stiffness and the external excitations are small; this is required for the asymptotic analysis of these equations, and is achieved by introducing again the small parameter $|\varepsilon| \ll 1$ of the previous section. Moreover, fundamental resonances of this system will be examined, i.e. steady state responses with frequencies identical to those of the excitation. To perform this analysis, the following additional requirement is imposed:

$$
\omega=1+\varepsilon \sigma
$$

where $\sigma$ is a 'detuning' parameter. Analysing the system using the method of multiple scales [44], the following approximations are obtained for its steady-state responses [43]:

$$
x_{1}(t)=a_{1} \cos \left(\omega t+\beta_{1}\right)+O(\varepsilon), \quad x_{2}(t)=a_{2} \cos \left(\omega t+\beta_{2}\right)+O(\varepsilon)
$$

where the amplitudes and phases are computed by solving the following set of algebraic equations:

$$
\begin{aligned}
0= & (-1 / 2) c_{1} a_{1}+(3 / 8) K a_{2} a_{1}^{2} \sin \phi-(3 / 8) K a_{1} a_{2}^{2} \sin 2 \phi+(3 / 8) K a_{2}^{3} \sin \phi-P_{1} \sin \beta_{1} \\
0= & (-1 / 2) c_{2} a_{2}-(3 / 8) K a_{1} a_{2}^{2} \sin \phi+(3 / 8) K a_{2} a_{1}^{2} \sin 2 \phi-(3 / 8) K a_{1}^{3} \sin \phi-P_{2} \sin \beta_{2} \\
0= & (3 / 8)(1+K) a_{1}^{3}+(3 / 8) K a_{1} a_{2}^{2} \cos 2 \phi-(9 / 8) K a_{2} a_{1}^{2} \cos \phi-(3 / 8) K a_{2}^{3} \cos \phi \\
& +(3 / 4) K a_{1} a_{2}^{2}-P_{1} \cos \beta_{1}-\sigma a_{1}
\end{aligned}
$$



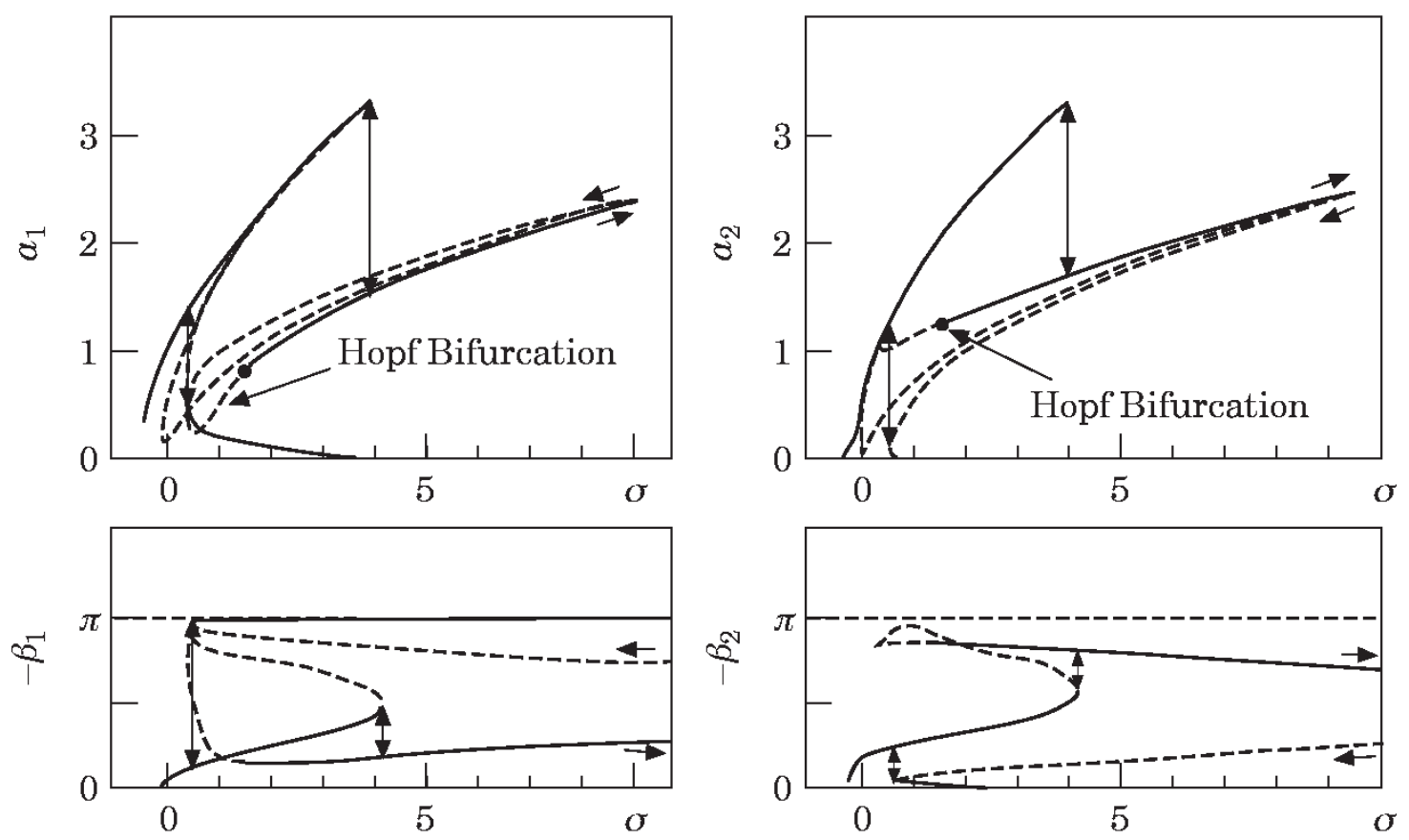

(b)
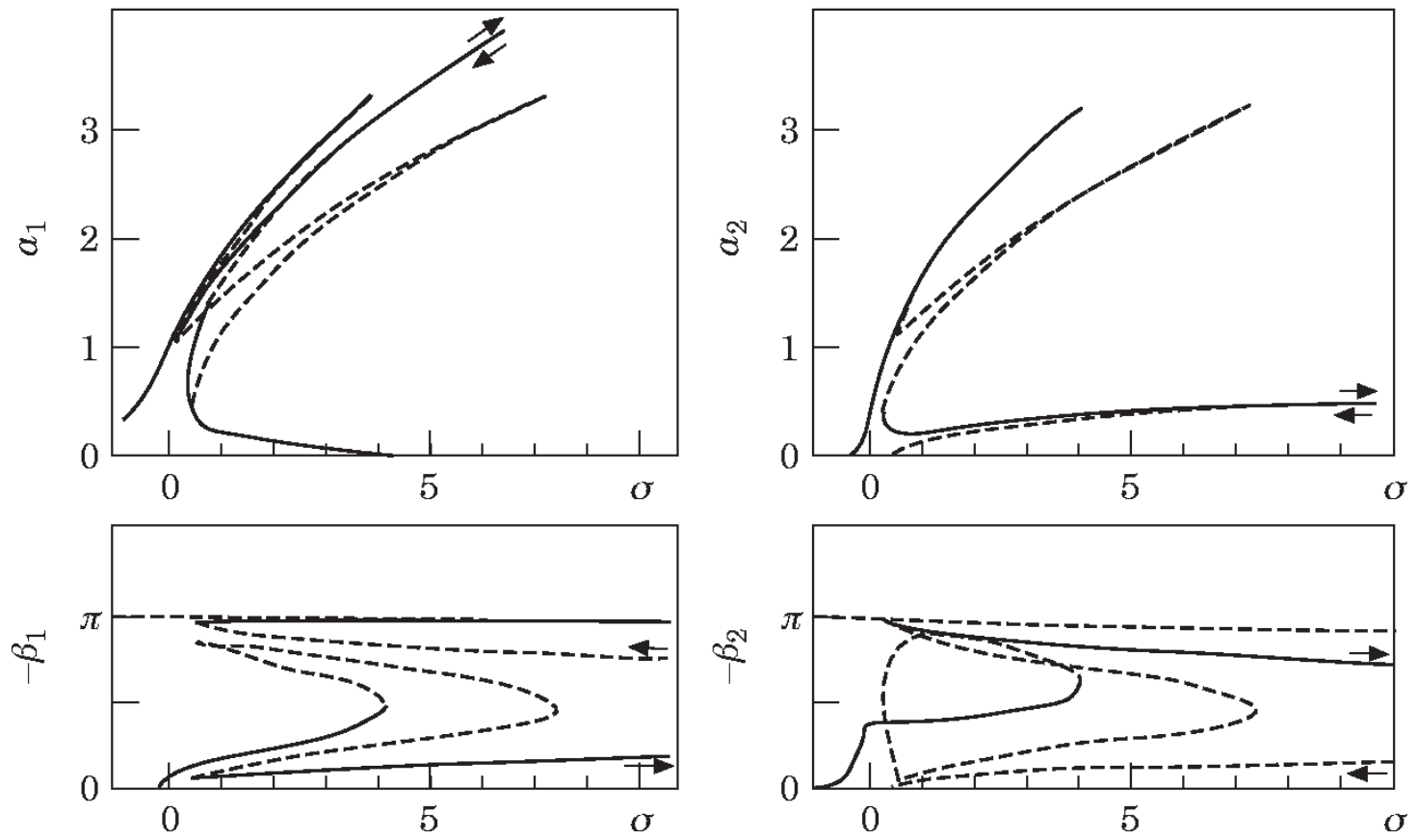

Figure 4. Fundamental resonance curves: (a) before the NNM bifurcation $(K=0.4>1 / 4)$; and (b) after the NNM bifurcation $(K=0.1<1 / 4)$ : — , stable; ---, unstable steady states.

$$
\begin{aligned}
0= & (3 / 8)(1+K) a_{2}^{3}+(3 / 8) K a_{2} a_{1}^{2} \cos 2 \phi-(9 / 8) K a_{1} a_{2}^{2} \cos \phi-(3 / 8) K a_{1}^{3} \cos \phi \\
& +(3 / 4) K a_{2} a_{1}^{2}-P_{2} \cos \beta_{2}-\sigma a_{2}
\end{aligned}
$$

These equations were numerically solved for parameters $c_{1}=0.05, c_{2}=0.07, P_{1}=0.2$ and $P_{2}=0$. A stability analysis was also performed.

As mentioned in the previous section, depending on the value of the coupling stiffness the system can possess two or four NNMs (in the later case one of these modes is unstable). Since the fundamental resonances occur in neighborhoods of NNMs, it is expected that the topological structure of the resonance curves of this system will crucially depend on the structure of its normal modes. This is evident from the results depicted in Fig. 4, where the fundamental resonance curves for a system with $K=0.4>1 / 4$ [Fig. 4(a); before the NNM bifurcation of Fig. 2] and $K=0.1<1 / 4$ [Fig. 4(b); after the NNM of Fig. 2] are 
depicted. For $K=0.4$ [Fig. 4(a)], the unforced system possesses two NNMs, and at most three stable steady-state solutions can exist at any value of the frequency detuning parameter $\sigma$. The steady states occur in the neighborhoods of the stable non-linear normal modes and for sufficiently large detuning values a stable-unstable pair of fundamental resonances exist close to each NNM. As discussed in [43] the branches of forced resonances can be considered as perturbations of the 'backbone curves' (amplitude-frequency relations for free oscillations) of the NNMs of the system. This is analogous to the linear case where steady-state resonance curves can be considered as perturbations of the constant-frequency straight lines eminating from the natural frequencies of the oscillator.

A change in the value of the parameter $K$ can introduce qualitative and quantitative changes in the topology of the fundamental resonances. For $K=0.1$ [Fig. 4(b)] an additional branch of steady-state solutions exists in the neighbourhood of one of the (stable) bifurcated modes (the one corresponding to large values of $a_{1}$ and small values of $a_{2}$ ). In this case, no stable steady solutions exist in the neighbourhoods of the unstable anti-phase mode, or of the bifurcated mode corresponding to large amplitudes $a_{2}$ (because only one of the masses is excited, and also due to damping).

These results show that: (i) the structure of forced resonances of non-linear oscillators depends crucially on the structure of their NNMs; and (ii) in non-linear systems, there may be more resonance branches than dof. Performing a mere linearised analysis and not taking into account essentially non-linear resonances due to unaccounted for NNMs may lead to inadequate modeling of the dynamics, and, as a result, to poor satisfaction of the performance objectives.

To illustrate better the previous points, a second example with a more practical nonlinear structure is presented. Consider the cyclic flexible assembly depicted in Fig. 5, consisting of $N$ identical uniform cantilever beams, coupled by means of linear stiffnesses. Assuming beam inextensionality and finite-amplitude oscillations, non-linear inertia and curvature terms give rise to geometric non-linearities which, as shown, can affect significantly the dynamic response. For an analysis of the forced vibrations of this system, the reader is referred to [39]. The governing equations of motion are of the following form:

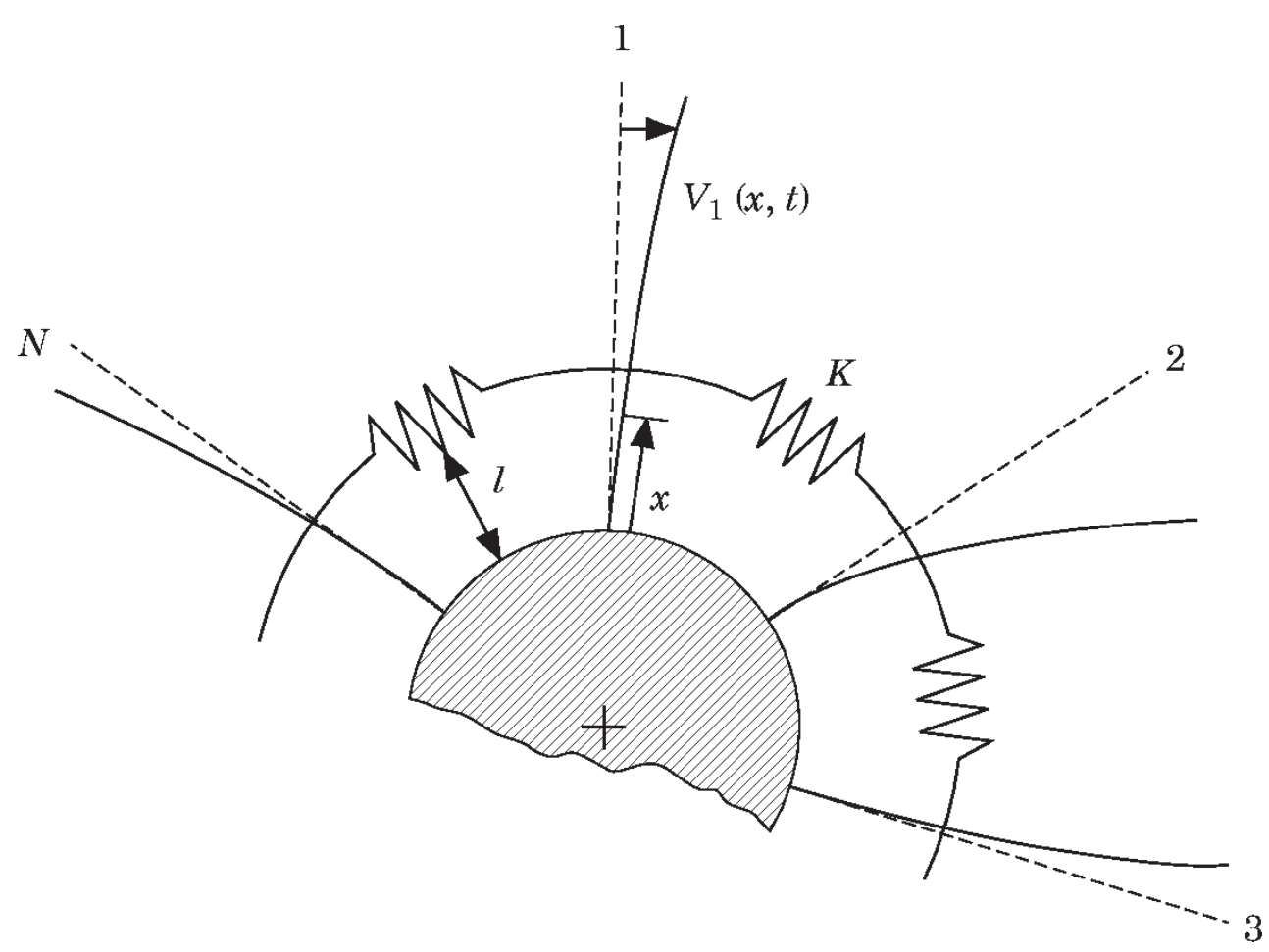

Figure 5. The cyclic assembly of $N$ geometrically non-linear beams. 


$$
\begin{aligned}
v_{i t t}+ & v_{i x x x x}+\varepsilon c_{i} v_{i t}+\varepsilon\left\{v_{i x}\left[v_{i x} v_{i x x}\right]_{x}+\frac{1}{2} \int_{1}^{x}\left[\int_{0}^{s} v_{i \xi}^{2}(\xi, t) \mathrm{d} \xi\right]_{t t} \mathrm{~d} s\right\}_{x} \\
= & -\varepsilon \frac{K L^{4}}{E I}\left\{2 v_{i}(1 / L)-v_{i-1}(1 / L)-v_{i+1}(1 / L)\right\} \delta(x-1 / L) \\
& +\frac{P_{i}(x, t)}{\varepsilon^{1 / 2}} \quad i=1, \ldots, N
\end{aligned}
$$

where $K$ is the non-linear coupling stiffness, $c_{i}$ is the coefficient of distributed viscous damping for the $i$ th beam, $\delta(\cdot)$ is Dirac's function, $P_{i}(x, t)$ is the distributed excitation applied to the $i$ th beam, and $v_{0} \equiv v_{N}, v_{N+1} \equiv v_{1}$ due to cyclicity. As previously, it is assumed that the coupling linear stiffnesses and the viscous damping coefficients are small, $O(\varepsilon)$ quantities (with $|\varepsilon| \ll 1$ ), and that the beam deflections are of $O\left(\varepsilon^{1 / 2}\right)$. It is additionally assumed that the distributed excitations $P_{i}(x, t)$ possess harmonic time dependence of frequency $\left(\omega_{1}+\varepsilon \sigma\right)$ and identical spatial distribution to the first linearised cantilever mode of each beam:

$$
P_{i}(x, t)=\Phi_{1}(x) \cos \left(\omega_{1}+\varepsilon \sigma\right) t, \quad 0<x<1, \quad i=1, \ldots, N
$$

In (22), $\Phi_{1}(x)$ is the spatial distribution and $\omega_{1}$ the natural frequency of the first linearised cantilever mode of each beam. Again consider fundamental resonances, and approximate the steady state responses of the beams as follows [39]:

$$
v_{i}(x, t)=\Phi_{1}(x) a_{i} \cos \left[(\omega+\varepsilon \sigma) t+\beta_{i}\right]+O(\varepsilon), \quad i=1, \ldots, N
$$

In Fig. 6 the fundamental resonance branches of the system with $N=4$ beams, and harmonic excitation applied to only beam 1 is shown. The coupling stiffness is assigned a value which guarantees the existence of additional NNMs with no counterparts in linear theory. The complicated structure of the resonance curves is caused by NNM bifurcations taking place in the unforced, undamped system, which increase in complexity as the number of beams increases. As seen from Fig. 6, as many as 12 stable co-existing fundamental resonances, exist; linear theory would predict the existence of, at most, four resonances.

\subsection{NON-LINEAR LOCALISATION AND MOTION CONFINEMENT}

One of the most interesting applications of NNMs in vibration analysis is non-linear mode localisation. This term means that the property of a subclass of NNMs is spatially confined, i.e. to localise to certain areas of a structure. For example, considering the NNMs of the 2-dof symmetric system depicted in Fig. 1, it can be seen that as the coupling stiffness decreases (i.e. for small $K$ ), the NNMs corresponding to small or large $c$ localise to either one of the masses. Hence, for sufficiently weak coupling stiffness, the oscillator of Fig. 1 possesses two 'spatially extended' modes (the in-phase and anti-phase NNMs), and two localised ones. Similarly, considering the resonance plots of Fig. 6 for the cyclic assembly, it is noted that branch 1 of fundamental resonances is strongly localised, since it corresponds to a response that is mainly confined to the directly forced beam 1. This strongly localised solution occurs in the neighbourhood of localised NNM of the undamped unforced system [39], and has no counterpart in linear theory. There also exist weakly localised resonance branches during which the forced beam and adjacent beams oscillate with comparable amplitudes, whereas all other beams undergo much smaller vibrations (branches 7 and 8, Fig. 6). Hence, a basic property of a subclass of NNMs is that they spatially confine vibrational energy, a feature which, as discussed later, can find application in vibration and shock isolation designs of mechanical oscillators. 
linear periodic systems. This is caused by spatial motion confinement of certain of their normal modes. Mode localisation can be beneficial in cases where a confinement of the vibrational energy is required, and many researchers developed analytical and computational techniques to study linear localisation of vibrational energy in disordered oscillators [46-51].

The concept of NNM provides a valuable tool for understanding mode localisation and motion confinement in non-linear vibration systems. In that context, non-linear mode localisation is defined as the spatial confinement of the vibrational energy of a subset of NNMs of the system. Previous authors studied localised buckling phenomena in forced non-linear elastic systems [52-54]. In the works by Vakakis and co-workers, non-linear localisation in discrete $[34,55,56]$ and continuous [38-42, 57-60] periodic systems with stiffness non-linearities were studied. A main result of these works is that, in contrast to linear periodic systems where structural disorder and weak substructure coupling are required for mode localisation, non-linear mode localisation can occur in perfectly symmetrical non-linear systems (i.e. with no disorder), and the only prerequisite for its existence is weak substructure coupling. To demonstrate this basic finding, a weakly coupled non-linear system with cyclic symmetry is considered [56].

Consider a discrete cyclic system similar to the continous structure depicted in Fig. 5 but with single dof substructures that are coupled by weak linear stiffnesses. Assuming weak cubic stiffness non-linearities for the grounding stiffness of each mass, the equations of motion are given by:

$$
\ddot{x}_{i}=-x_{i}-\varepsilon \mu x_{i}^{3}-\varepsilon k\left(x_{i}-x_{i+1}\right)-\varepsilon k\left(x_{i}-x_{i-1}\right), \quad i=1, \ldots, N
$$

In [56], the NNMs of this system were asymptotically studied using the method of multiple scales. The system admits NNMs of the following form:

for $N=(2 p+1)$ :

$$
\begin{gathered}
x_{1}(t)=a_{1} \cos \left[(1+\varepsilon \alpha) t+\beta_{1}\right]+O(\epsilon) \\
x_{2}(t)=x_{N-1}(t)=-a_{2} \cos \left[(1+\varepsilon \alpha) t+\beta_{1}\right]+O(\varepsilon) \\
x_{3}(t)=x_{N-2}(t)=a_{3} \cos \left[(1+\varepsilon \alpha) t+\beta_{1}\right]+O(\varepsilon) \\
\ldots \\
x_{p+1}(t)=x_{p+2}(t)=(-1)^{p} a_{p+1} \cos \left[(1+\varepsilon \alpha) t+\beta_{1}\right]+O(\varepsilon)
\end{gathered}
$$

for $N=2(p-1)$ :

$$
\begin{gathered}
x_{1}(t)=a_{1} \cos \left[(1+\varepsilon \alpha) t+\beta_{1}\right]+O(\varepsilon) \\
x_{2}(t)=x_{N-1}(t)=-a_{2} \cos \left[(1+\varepsilon \alpha) t+\beta_{1}\right]+O(\varepsilon) \\
x_{3}(t)=x_{N-2}(t)=a_{3} \cos \left[(1+\varepsilon \alpha) t+\beta_{10}\right]+O(\varepsilon) \\
\ldots \\
x_{p-1}(t)=x_{p+1}(t)=(-1)^{p} a_{p-1} \cos \left[(1+\varepsilon \alpha) t+\beta_{1}\right]+O(\varepsilon) \\
x_{p}(t)=(-1)^{p+1} a_{p} \cos \left[(1+\varepsilon \alpha) t+\beta_{1}\right]+O(\varepsilon)
\end{gathered}
$$

where the phase $\beta_{1}$ is determined by the initial conditions, and $\varepsilon \alpha=\varepsilon \alpha\left(a_{1}\right)$ is the small amplitude-dependent non-linear correction to the frequency of oscillation. The ratios of the amplitudes $\left(a_{i} / a_{j}\right)$ were determined in [56] for systems with various dof. The localised NNMs for the systems with $N=4$ and 5 dof are presented in Fig. 7(a), (b). It turns out that, for a fixed level of energy, the structural parameter that controls the degree of 
localisation in the system is the ratio $(k / \mu)$, i.e. the ratio of coupling to grounding non-linearity. For low values of this ratio, $(k / \mu) \leqslant 0.1$, non-linear mode localisation occurs and the vibrational energy is mainly confined in mass 1 ; this is despite direct coupling between masses. As $(k / \mu)$ increases, the localised branches of the system with $N=4$ [(Fig. 4(a)] become non-localised and eventually coalesce with the antiphase non-localised NNM $a_{1}=a_{2}=a_{3}=a_{4}$ in a pitchfork bifurcation. For the system with $N=5$, as the ratio $(k / \mu)$ increases, the localised NNM becomes non-localised following a continuous branch of solutions and no mode bifurcations occur.

The previous results show that the non-linear cyclic system possesses localised NNMs where the vibrational energy is passively confined mainly to one mass. Non-linear localisation in a structure can greatly influence its transient response since it can lead to passive motion confinement of disturbances generated by external forces: in such a structure, motions generated by external impulsive excitations remain passively confined close to the point where they are initially generated instead of 'spreading' through the
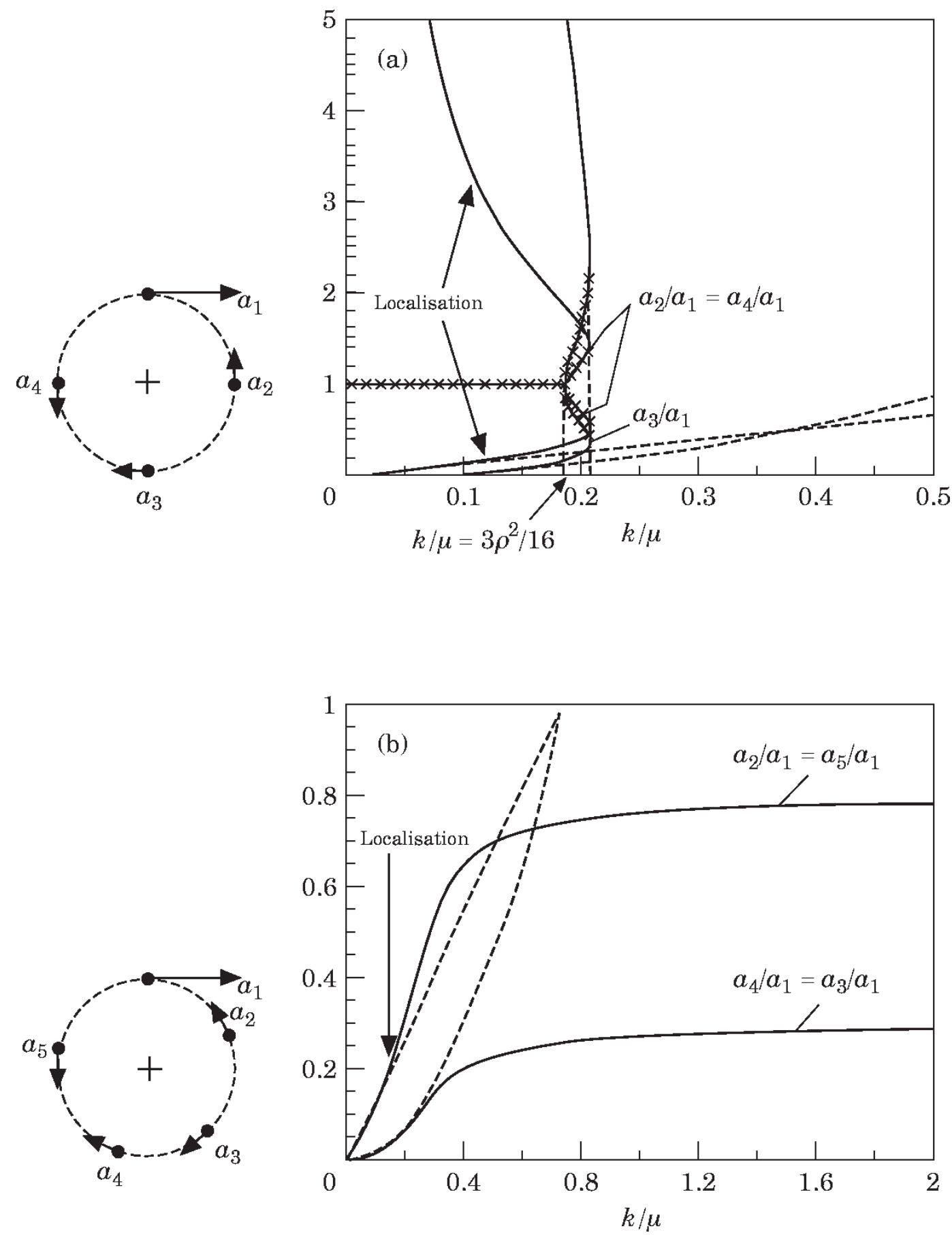

Figure 7. Non-linear localisation in a cyclic system with, (a) $N=4$ and (b) $N=5$ masses. — $* * *$, unstable NNMs. 


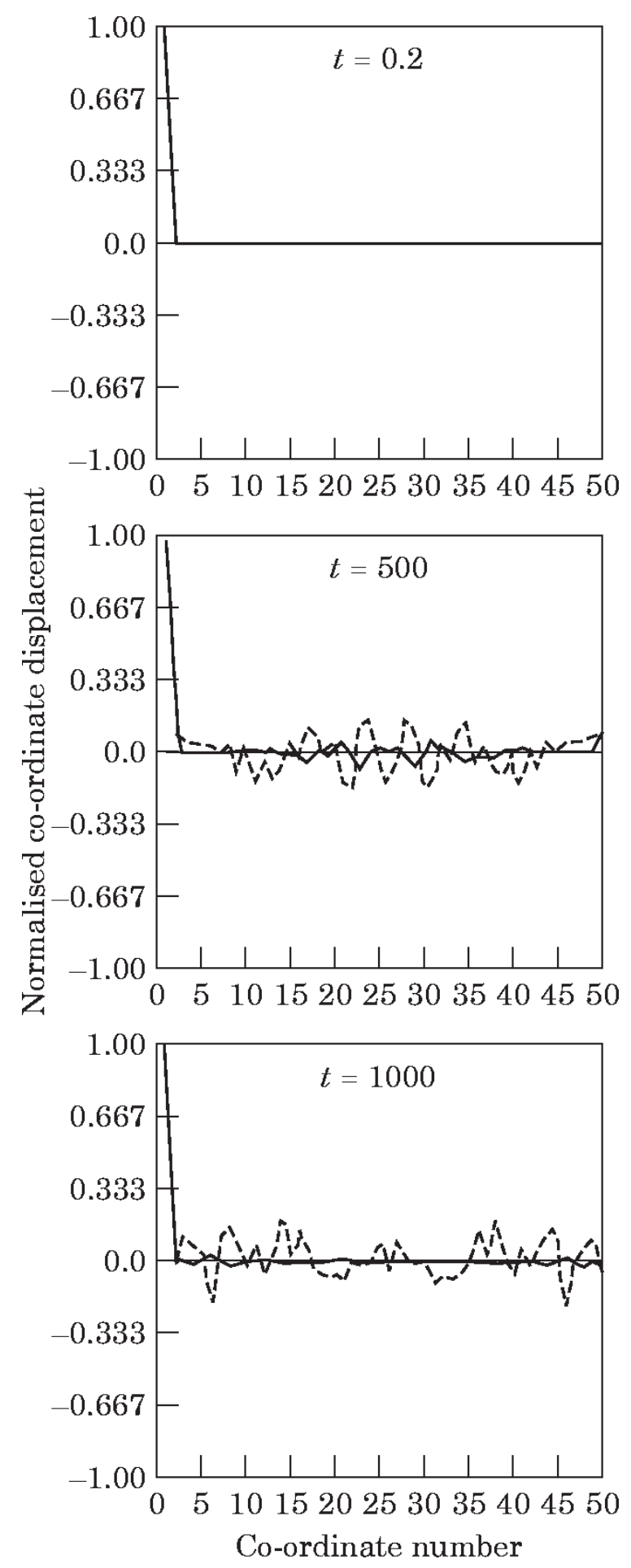

Figure 8. Transient passive motion confinement in the system with 50 dof, impulse applied at mass 1. Non-linear system; ---, linear system.

entire structure. Such a passive confinement phenomenon in perfectly symmetric cyclic systems has no counterpart in linear theory, where energy is transferred from one mass to another in the well-known beat phenomenon.

To demonstrate the passive non-linear motion confinement phenomenon, consider the impulsive response of the previous discrete cyclic system. As shown previously, as $(k / \mu) \rightarrow 0$ a branch of NNMs localises strongly to a single mass of the system. A system with $N=50$ masses is considered and the numerical results are obtained by finite element computations [56]. A force with unit magnitude is applied for a duration of $\Delta t=0.2 \mathrm{~s}$ at mass 1 , and in Fig. 8 the transient responses of the cyclic system with parameters $\varepsilon \mu=0.3, \varepsilon k=0.05$, and ratio $k / \mu=0.166$ (weakly coupled system) are depicted, at time instants $t=0.2,500$, and $1000 \mathrm{~s}$. In these plots, the instantaneous normalised displacements of all masses of the system are presented. For comparison purposes, the responses of the corresponding linear system with $\varepsilon \mu=0$ are also shown in these graphs. Clearly, in the non-linear case the 
energy is mainly confined to mass 1 instead of 'leaking' to the entire system; by contrast, in the linear case there is a gradual 'spreading' of the injected energy to all masses of the system. This spreading of energy in the linear system becomes more profound as time increases.

The passive transient motion confinement is attributed to the stable localised NNM of the system, which, once excited by the impulse, restricts the propagation of energy through the entire system. Hence, localised NNMs can be a novel tool for vibration and shock isolation designs of mechanical systems, since a system whose inherent dynamics leads to motion confinement of external disturbances is more amenable to active or passive control than a system with no such dynamical properties. In a recent work [61], non-linear localisation was employed to design and optimise a vibration isolator that suppresses disturbances generated by a spinning momentum flywheel in a spacecraft application; this suppression is achieved in wide frequency ranges.

\subsection{ADDITIONAL APPLICATIONS}

NNMs can be used in additional areas of vibration theory. Starting from the area of modal analysis and system identification (MA-SI), traditional techniques for analysing non-linear structures are based on the assumptions of weak non-linearities and of a modal structure similar to that of an underlying linearised system. As shown above, even a simple 2-dof system can have more normal modes than its dof; hence, in performing non-linear MA-SI, one must consider the possibility that certain of the sought modes are essentially nonlinear, with no counterparts in linear theory. The localised NNMs discussed in Section 3.2 are a subclass of such modes with no linear counterparts. In that context, the concept of NNM can provide a valuable tool for understanding the effects of structural non-linearities on the dynamics, and for developing a new class of non-linear MA-SI methodologies that can be used for analysing practical structures with essential non-linearities, such as clearances or dry friction.

In some recent experimental works [59,60,62], the existence of the theoretically predicted transient and steady-state non-linear localisation in a flexible assembly of beams was verified. The localisation phenomenon can be implemented in novel active or passive vibration isolation designs, where a disturbance generated by external forces is first spatially confined to a predetermined part of a structure, and then, passively/actively eliminated. Inducing localised NNMs in a flexible structure enhances its controllability, since in designing for active control one needs to consider only a small substructure (where the disturbance is confined) instead of controlling the entire structure. Of course, issues of control spill-over and excitation of unwanted modes should also be addressed. In addition, the study of motion confinement phenomena due to non-linearities can be beneficial in applications where such localisation phenomena are unwanted. For example, localisation of vibrational energy in rotating turbine blade assemblies can be catastrophic since it may lead to failure of highly rotating blades [63]. Understanding the mechanisms (such as, structural disorder and non-linearities) that lead to motion confinement can prevent such failures and prolong the operational life of mechanical components. To give an indication of the diverse applications of non-linear localization, Achong [64] showed that non-linear localisation can explain certain dynamic phenomena in a steelpan musical instrument.

As an example of localisation induced by non-linear effects, consider the experimental fixture of Fig. 9 consisting of two identical cantilever beams. Direct coupling between the beams is realised using an elastic element and rigid constraints are used to induce clearance non-linearities; the resulting vibro-impact oscillations were analysed experimentally in [62] and only a brief summary of results is provided here. Clearance non-linearities of the type 
considered in the fixture of Fig. 9 are very common in engineering structures, resulting from loose joints or from impacting vibrating members. In Fig. 10, the tip accelerations of the two beams during vibro-impact oscillations are shown. Single-side impacts were considered by removing one of the two rigid barriers of each beam. An initial displacement is given to beam 1 and the ensuing transient response of the system is studied. For comparison purposes, Fig. 11 depicts the corresponding linear responses of the beams (i.e. when the rigid barriers are removed). Comparing Figs. 10 and 11, it is clear that in the system with vibro-impacts, non-linear localisation occurs in the directly excited beam with only a small portion of the vibrational energy 'leaking' to the unexcited beam. This is in contrast to the linear case where transfer of energy between the two beams takes place in the well-known beat phenomenon. As numerical simulations indicate [62] this localisation phenomenon is due to the clearance non-linearities and is not affected by the structural damping of the beams or by the energy dissipated due to inelastic impacts at the rigid barriers.

On a theoretical level, the concept of NNMs can be employed to analyse the dynamics of discrete and continuous non-linear oscillators, and to identify new dynamic phenomena (such as the non-linear motion confinement phenomenon discussed above). In particular, Nayfeh and co-workers [30,31,65] introduced complex NNM manifolds and studied NNM bifurcations in discrete systems. The conceivement of non-linear superposition principles would be of interest to express general transient responses as non-linear superpositions of individual NNM responses; this could be considered as a non-linear analogy of the principle of linear superposition of the classical linear vibration theory.

Finally, as shown in the works by Shaw [66] and Shaw and Pierre [25-28], the NNMs of elastic structures can be used as the basis for Gallerkin procedures that reduce the number of dof required for the dynamic analysis (model reduction). It was found that a basis consisting of NNMs can capture more accurately the structural dynamics than a similar basis composed of linearised modes. Hence, NNMs can be also used for order

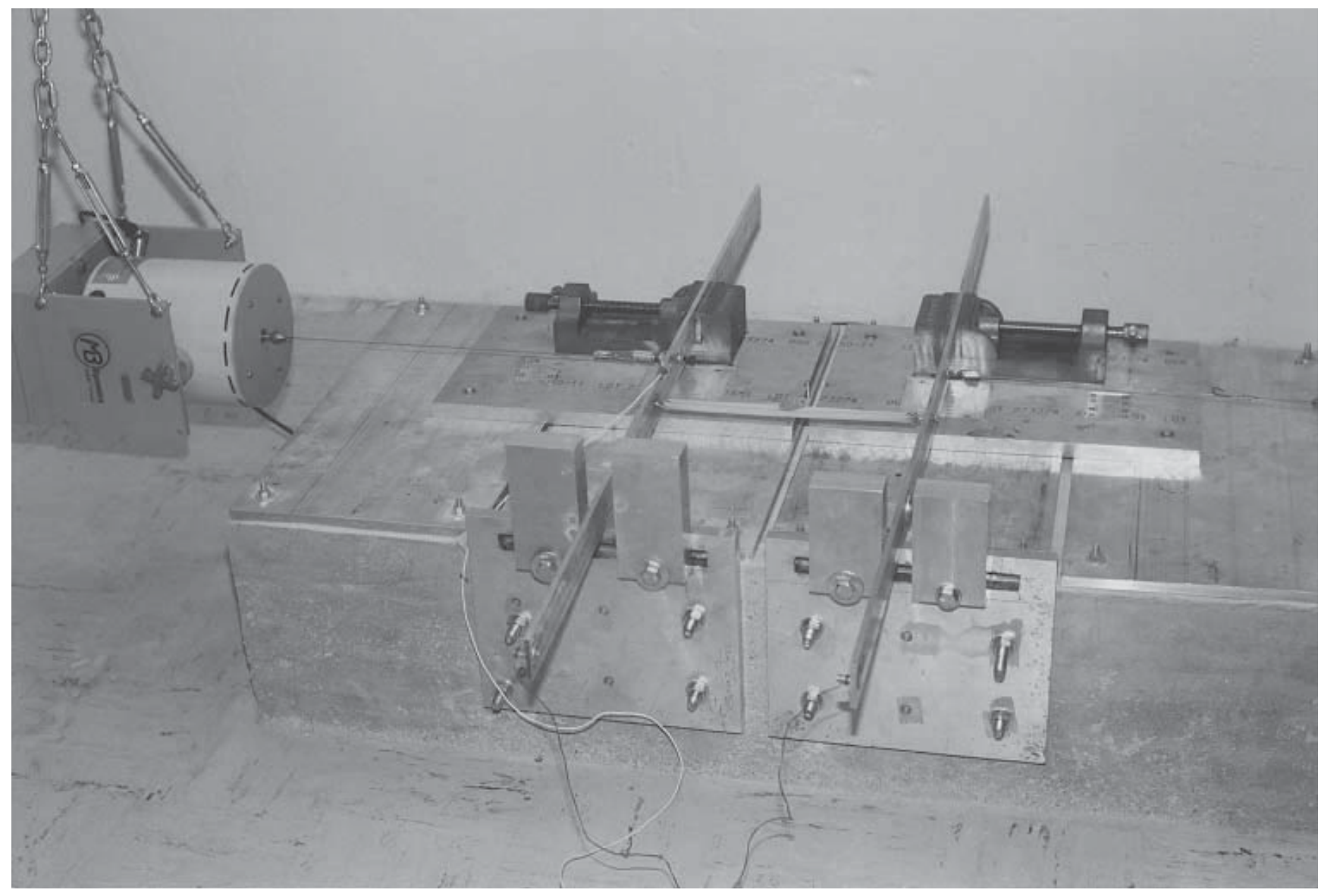

Figure 9. Experimental fixture for studying non-linear localisation due to vibro-impacts. 

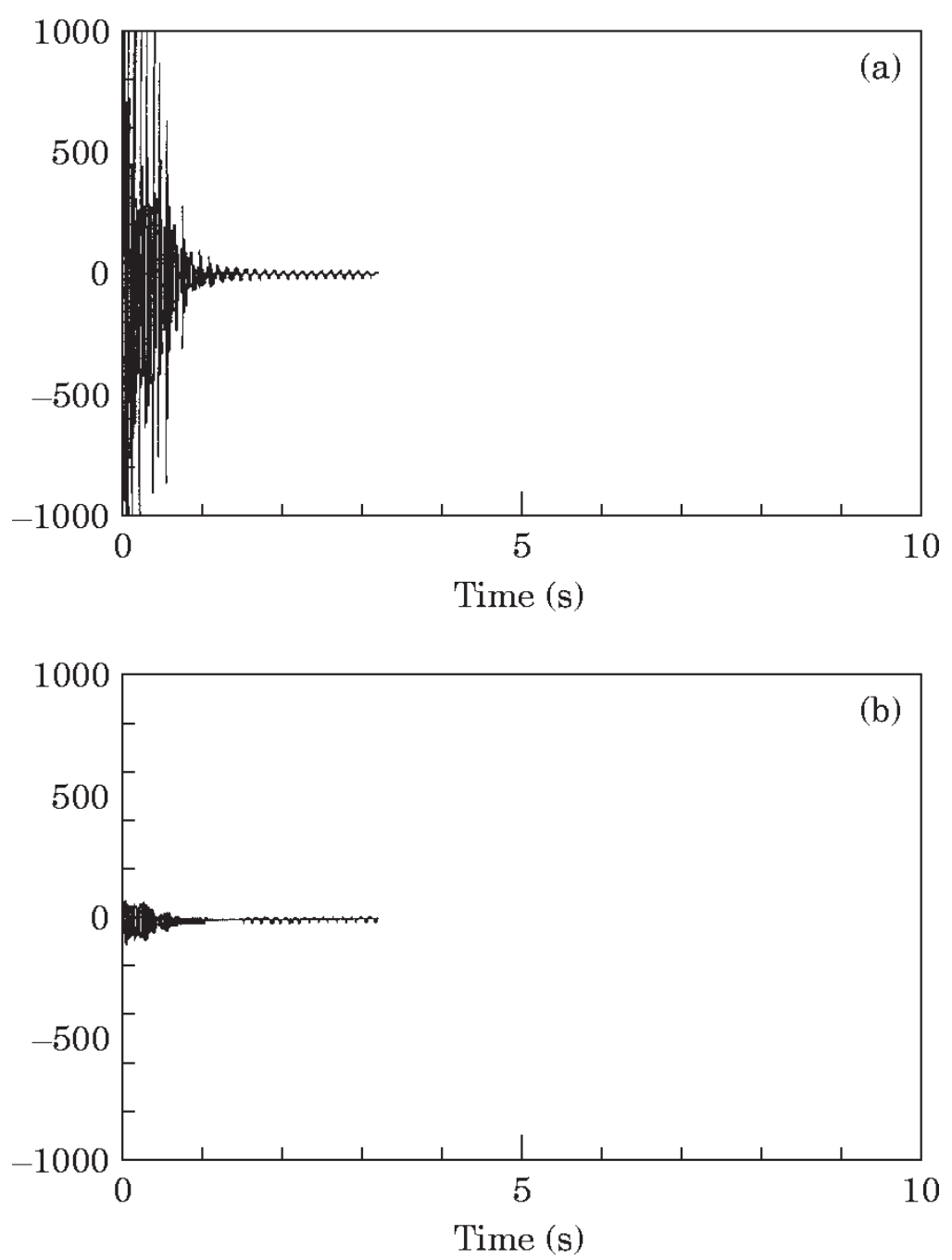

Figure 10. Transient non-linear motion confinement: tip acceleration of (a) the directly excited beam 1, and (b) the unexcited beam 2 .

reduction of non-linear systems. In the aforementioned works, a new non-linear modal analysis method based on NNMs that 'decouples' the equations of motion of non-linear oscillators was also derived.

\section{CONCLUSIONS}

An NNM has been defined, and some of its applications in vibration theory have been examined. All engineering structures in nature are inherently non-linear, the non-linearities being kinematic, material, and geometric. For example, in the case of a rotating blade disc assembly, a dynamic unbalance or a pressure differential in the surrounding fluid may excite structural resonances leading to large scale motions where non-linearities are strongly affecting the response. Similarly, large scale motions of truss structures may be strongly influenced by clearance non-linearities of the joints connecting individual components, or by geometric non-linearities arising from the relatively large deflections of oscillating structural members. In such cases, the concept of NNM can be used to understand and model non-linear dynamic phenomena that might have no counterparts in linear theory. Ignoring the non-linear effects and using linear modes to model the dynamics, although this might work in some cases, could leave essential dynamics unmodelled which could significantly influence the overall response. In cases where the non-linearities are profound, one should consider non-linear concepts for carrying out the dynamic analysis, such as NNMs. 
15. D. G. Magiros 1961 Journal of Mathematical Physics, 2, 869-875. Method for defining principal modes of nonlinear systems utilizing infinite determinants.

16. R. H. RAND 1971 Journal of Applied Mechanics, 38, 561. Nonlinear normal modes in two degrees of freedom systems.

17. R. H. RAND 1971 International Journal of Nonlinear Mechanics 6, 545-547. A higher order approximation for nonlinear normal modes in two degrees of freedom systems.

18. R. H. RAND 1973 International Journal of Nonlinear Mechanics 8, 161-168. The geometrical stability of NNMs in two dof systems.

19. R. H RAND 1974 International Journal of Nonlinear Mechanics 9, 363-368. A direct method for NNMs.

20. R. H. RAnd and R. Vito 1972 Journal of Applied Mechanics 39, 296-297. Nonlinear vibrations of two degree of freedom systems with repeated linearized natural frequencies.

21. L. I. Manevitch and Yu. V. Mikhlin 1972 PMM 36(6), 1051-1058. On periodic solutions close to rectilinear normal vibration modes.

22. L. I. Manevitch and V. N. Pilipchuk 1981 Prikl. Mech. 13, 97-103. Nonlinear vibrations of a three-degree-of-freedom mechanical system with several equilibrium states.

23. Yu. V. Mikhlin 1985 Pikladneya Matematika i Mekhanika 49, 738-743. The joining of local expansions in the theory of nonlinear oscillations.

24. T. K. Caughey and A. F. Vakakis 1991 International Journal of Nonlinear Mechanics 26, 89-103. A method for examining steady state solutions of forced discrete systems with strong nonlinearities.

25. S. W. ShaW and C. Pierre 1991 Journal of Sound and Vibration 150, 170-173. Nonlinear normal modes and invariant manifolds.

26. S. W. Shaw and C. Pierre 1992 On nonlinear normal modes. In: Nonlinear Vibrations. Mexico.

27. S. W. Shaw and C. Pierre 1993 Journal of Sound and Vibration 164, 85-124. Normal modes for nonlinear vibratory systems.

28. S. W. Shaw and C. PIERre 1994 Journal of Sound and Vibration, in press. Normal modes of vibration for nonlinear continuous systems.

29. N. Boivin, C. Pierre and S. W. Shaw 1993 Nonlinear normal modes, invariance, and modal dynamics approximations of nonlinear systems. In: Nonlinear Vibrations. Mexico.

30. A. H. NAYFeH and S. A. NAYFeH 1993 Journal of Vibration and Acoustics 117, 199-205. Nonlinear normal modes of a continuous system with quadratic nonlinearities.

31. A. H. NAYfeh and S. A. NAYFeH 1994 Journal of Vibration and Acoustics 116, 129-136. On nonlinear modes of continuous systems.

32. A. H. Nayfeh, J. F. Nayfeh and D. T. Mook 1992 Nonlinear Dynamics 2, 145-162. On methods for continuous systems with quadratic and cubic nonlinearities.

33. M. Pakdemirli and A. H. NAYFeH 1997 Journal of Vibration and Acoustics, in press. Nonlinear vibrations of a beam-spring-mass system.

34. A. F. VAKAKIS 1992 Journal of Sound and Vibration 158, 341-361. Nonsimilar normal oscillations in a strongly nonlinear discrete system.

35. G. S. Happawana, A. K. Bajaj and M. Axene 1995 Journal of Sound and Vibration 183, 361-368. Analytical solution to nonsimilar normal modes in a strongly nonlinear discrete system.

36. A. H. NAYfeH and C. Chin 1993 Journal of Vibration and Acoustics, in press. On nonlinear normal modes of systems with internal resonance.

37. M. E. KING and A. F. VAKakis 1993 Journal of Vibration and Acoustics 116, 332-340. An energy-based formulation for computing nonlinear normal modes in undamped continuous systems.

38. M. E. KING and A. F. VaKakis 1994 Wave Motion 19, 391-405. A method for studying waves with spatially localized envelopes in a class of weakly nonlinear partial differential equations.

39. M. E. KING and A. F. VAKaKis 1995 Nonlinear Dynamics 7, 85-104. A very complicated structure of resonances in a nonlinear system with cyclic symmetry.

40. M. E. KING and A. F. VAKakis 1995 ZAMM 75, 127-139. Mode localization in a system of coupled flexible beams with geometric nonlinearities.

41. M. E. KING and A. F. VAKAKIS 1995 International Journal of Solids and Structures 32, 1161-1177. Asymptotic analysis of nonlinear mode localization in a class of coupled continuous structures.

42. A. F. Vakakis and M. E. KING 1995 Journal of Acoustic Society of America 98, 1534-1546. Nonlinear wave transmission in a mono-coupled elastic periodic system.

43. A. F. VAKakis 1992 Nonlinear Dynamics 3, 123-243. Fundamental and subharmonic resonances in a system with a ' $1-1$ ' internal resonance. 
44. A. H. Nayfeh and D. T. Mook 1984 Nonlinear Oscillations. New York: John Wiley.

45. P. W. Anderson 1958 Physics Reviews 109, 1492-1505. Absence of diffusion in certain random lattices.

46. C. Pierre and E. H. Dowell 1987 Journal of Sound and Vibration 114, 549-564. Localization of vibrations by structural irregularity.

47. S. T. WeI and C. PIERre 1988 Journal of Vibration and Acoustics 110, 429-438. Localization phenomena in mistuned assemblies with cyclic symmetry. Part I: free vibrations.

48. C. H. Hodges 1982 Journal of Sound and Vibration 82(3), 411-424. Confinement of vibration by structural irregularity.

49. 0. 0. BANDIKSEN 1987 AIAA Journal 25(9), 1241-1248. Mode localization phenomena in large space structures.

50. A. J. Keane and W. G. Price 1989 Journal of Sound and Vibration 128, 423-450. On the vibrations of mono-coupled periodic and near-periodic structures.

51. A. LuOngo 1992 Journal of Sound and Vibration 155, 249-271. Mode localization by structural imperfections in one-dimensional continuous systems.

52. J. M. T. Thompson and L. N. Virgin 1988 Physics Letters A126, 491-496. Spatial chaos and localization phenomena in nonlinear elasticity.

53. G. W. Hunt, H. M. Bolt and J. M. T. Thompson 1989 Proceedings of the Royal Society of London A425, 245-267. Structural localization phenomena and the dynamical phase-space analogy.

54. G. W. Hunt and M. K. WadeE 1991 Proceedings of the Royal Society of London A434, 485-502. Comparative Lagrangian formulations for localized buckling.

55. A. F. Vakakis and C. Cetinkaya 1993 SIAM Journal of Applied Mathematics 53, 265-282. Mode localization in a class of multi degree of freedom nonlinear systems with cyclic symmetry.

56. A. F. VAkakis, T. A. Nayfeh and M. E. King 1993 Journal of Applied Mechanics 60, 388-397. A multiple scales analysis of nonlinear, localized modes in a cyclic periodic system.

57. A. F. VAKakis 1994 AIAA Journal 32, 1902-1910. Passive spatial confinement of impulsive responses in coupled nonlinear beams.

58. M. E. King 1994 Ph.D. Thesis, University of Illinois at Urbana-Champaign, Urbana, Illinois. Analytical and Experimental Aspects of Nonlinear Normal Modes and Nonlinear Mode Localization.

59. M. E. King, J. A. S. Aubrecht and A. F. Vakakis 1995 Journal of Nonlinear Science 5, 485-502. Experimental nonlinear steady-state localization in coupled beams with active nonlinearities.

60. J. A. S. Aubrecht, A. F. Vakakis, T. C. Tsao and J. Bentsman 1996 Journal of Sound and Vibration, in press. Experimental study of nonlinear transient motion confinement in a system of coupled beams.

61. T. A. Nayfeh, E. Emaci and A. F. Vakakis 1997 AIAA Journal, in press. Application of nonlinear localization to the optimization of a vibration isolation system.

62. E. Emaci, T. A. Nayfeh and A. F. Vakakis 1997 Zeit. Ang. Math. Mech. (ZAMM), in press. Numerical and experimental study of nonlinear localization in a flexible structure with vibro-impacts.

63. W. G. Brown 1993 Proceedings of the 11th IMAC, Orlando, FL. Buffeting response of a bladed disc assembly.

64. A. Achong 1996 Journal of Sound and Vibration 197, 471-487. The steelpan as a system of nonlinear mode localized oscillators, part I: Theory, simulations, experiments and bifurcations.

65. A. H. NAyfeh, C. Chin and S. A. NAYFeH 1995 Journal of Vibration and Acoustics 117, 477-481. Nonlinear normal modes of a cantilever beam.

66. S. W. SHaw 1994 Journal of Nonlinear Science 4, 419-448. An invariant manifold approach to nonlinear normal modes of oscillation. 